\title{
Ausscheidungsprodukte von Hydroxyanthrachinonen im Harn bei Ratten
}

\author{
Von B. MähNer und H.-J. Dulce \\ Aus dem Institut für Angewandte Physiologische Chemie und Klinische Chemie der Freien Universität Berlin \\ (Direktor: Prof. Dr. H.-J. Dulce)
}

(Eingegangen am 29. Juni 1967)

Bei Ratten läßt sich nach Alizarinverfütterung aus dem Harn eine Fraktion isolieren, die zu ctwa $85 \%$ aus Alizarinmonoglucuronid bestcht. Das Alizarinmonoglucuronid ẉurde durch quantitativen Nachweis der Hydroxyanthrachinon- und Glucuronsäurekomponente und durch chromatographischen Nachweis der Spaltung mit $\beta$-Glucuronidase identifiziert.

Ruberythrinsäure wird bei Ratten nach oraler Gabe ebenso wie Alizarin als Alizarinmonoglucuronid im Harn ausgeschieden. Dies konntc durch den Nachweis der Identität der beiden nach Alizarin- und nach Ruberythrinsäureverfütterung aus dem Harn isolierten Hydroxyanthrachinonderviate bewiesen werden.

Innerhalb von 24 Stdn. findet man bei Ratten etwa 10\% dex zugeführten Alizarinmenge als Glucuronid im Harn wieder, bei Ruberythrinsäurezufuhr ctwa 8\%. Das Maximum der Ausscheidung der beiden Substanzen liegt etwa 6 Stdn. nach einmaliger oraler Zufuhr.

After feeding alizarin to rats, a fraction was isolated from the urine, which consisted of about $85 \%$ alizarin monoglucuronide. The alizarin monoglucuronide was identified by the identification and determination of the hydroxyanthraquinone and the glucuronic acid components, and by the chromatographic demonstration of the cleavage with $\beta$-glucuronidase.

Ruberythric acid is also excreted in the urine as alizarin monoglucuronide after its oral administration to rats; this was evident from the identity of the isolated hydroxyanthraquinone after feeding either alizarin or ruberythric acid.

Within $24 \mathrm{hr}$ of administering alizarin or ruberythric acid to rats, $10 \%$ or $8 \%$ respectively can be recovered from the urine as alizarin glucuronide. The maximum excretion from both compounds occurs at about $6 \mathrm{hr}$ after a single oral dose.

Die Hydroxyanthrachinone Alizarin (I) und Ruberythrinsäure (II) vermindern in vitro in einem Bereich oberhalb $\mathrm{pH}$ 6,8 die Kristallisation von Calciumoxalat und Calciumphosphat (1). Beide Hydroxyanthrachinone sind neben anderen substituierten Anthrachinonen aus der Krappwurzel (Wurzel der Färberröte, Rubia tinctoria) darstellbar.
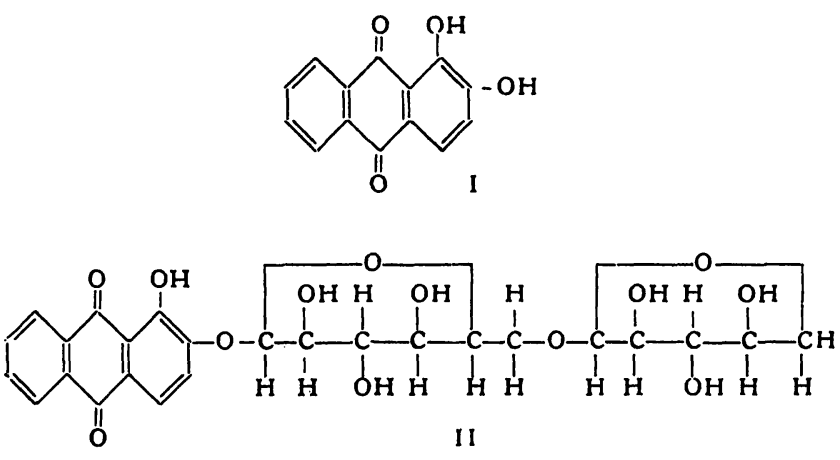

Krappwurzelextrakt verringert in vivo nach oraler Gabe die Bildung von calciumhaltigen Harnsteinen $(2,3)$. Unklarheiten herrschten bisher über die Ausscheidungsprodukte der beiden Hydroxyanthrachinone. Alizarin könnte als Glucuronid ausgeschieden werden, wie dies bei Phenolen häufig der Fall ist. Für die Ruberythrinsäure nahm $B_{A U E R}(4,5)$ an, daß sie unverändert eliminiert wird und dadurch eine Säuerung des Harnnes hervorruft.

Wir prüften deshalb im Tierversuch, in welcher Form Alizarin und Ruberythrinsäure nach oraler Zufuhr tatsächlich ausgeschieden und welche Menge an Stoffwechselprodukten der Hydroxyanthrachinone nach einmaliger oraler Gabe innerhalb von $24 \mathrm{Stdn}$. im Harn wiedergefunden werden.

\section{Methodik}

Isolierung der im Harn ausgeschiedenen Hydroxyanthrachinone

Etwa $260 \mathrm{~g}$ schweren männlichen Ratten wurde Alizarin bzw. Ruberythrinsäure (etwa $100 \mathrm{mg} / \mathrm{kg}, \mathrm{pH} 8,0$ ) mittels Schlundsonde verfüttert. Jeweils 10 Ratten hielten wir in einem StoffwechselKäfig, sammelten den Harn stdl. 6 Stdn. lang und bewahrten ihn bei $-18^{\circ}$ auf. Der Harn wurde nach Filtrieren mit $0,1 \mathrm{~N} \mathrm{HCl}$ auf $\mathrm{pH} 4$ angesäuert, anschließend zentrifugiert und zur präparativen Trennung der Úberstand auf Chromatographiepapier Schleicher und Schüll 2043b aufgetragen. Als Fließmittel verwendeten wir n-Propanol/Äthylacetat/ $\mathrm{H}_{2} \mathrm{O}$ 4:3:2 (v/v) (6). Die Laufzeit der Chromatogramme betrug $10 \mathrm{Stdn}$. Die sich abgrenzende gelb-rote Bande wurde mit Wasser eluiert, das Eluat im Rotationsverdampfer eingedampft und mit absolutem, unvergällten Alkohol ausgeschüttelt. Dabei ergaben sich zwei Fraktionen, eine in Alkohol unlösliche rot gefärbte Substanz und ein in Alkohol gelb gelöster Anteil. Die unlösliche Fraktion wurde abzentrifugiert, in Wasser erneut gelöst, abermals im Rotationsverdampfer getrocknet, und mit Alkohol ausgeschüttelt. Der nunmehr unlösliche Teil ergab nach Abzentrifugieren und Verdunsten des Alkohols die Fraktion I. Der im Alkohol lösliche Anteil ergab mehrfach mit Aktivkohle gereinigt und im Rotationsverdampfer eingeengt die Fraktion II.

Identifizierung der ausgeschiedenen Hydroxyanthrachinone

Photometrischer Glucuronsäure- und Alizarin-Nacbuveis

Fraktion I und II, gewonnen nach Alizaringabe, sowie Fraktion I, gewonnen nach Ruberythrinsäuregabe, wurden auf ihren Gehalt an Glucuronsäure und Alizarin untersucht. Glucuronsäure bestimmten wir mit Carbazol nach Dische (7) und Alizarin nach Spaltung der Fraktionen mit $\beta$-Glucuronidase (E. C. 3.2.1.31, $\beta$-D-Glucuronid Glucuronohydrolase) und Verdünnen mit $0,1 \mathrm{M}$ GlycinPuffer nach Sörensen pH 11 photometrisch bei $578 \mathrm{~nm}$. Eichkurven erhielten wir durch Messen der Verdünnungsreihen von Glucuronsäurelacton und Alizarin.

\section{Spaltung von Fraktion I mit $\beta$-Glucuronidase}

Die nach Alizarin- wie auch Ruberythrinsäuregabe isolierte Fraktion I $(11,4 \mathrm{mg})$ wurde in $30 \mathrm{~m} / 0,2 \mathrm{M}$ Acetatpuffer $\mathrm{pH} 5$ gelöst und mit je $0,2 \mathrm{~m} /$ Ketodase ( $\beta$-Glucuronidase Gocdecke $50000 \mathrm{E} / \mathrm{m} /$ ) 
in 10 Einzelansätzen von $3,0 \mathrm{~m} /$ bei $37^{\circ}$ inkubiert. In Abständen von je $1 \mathrm{Min}$. wurde in je einem Ansatz durch Zugabe von $0,3 \mathrm{~m} l$ $2 \mathrm{~N} \mathrm{NaOH}$ die Spaltung unterbrochen und nach Zugabe von $3 \mathrm{~m} /$ $0,1 \mathrm{M}$ Glycin-Puffer $\mathrm{pH} 11$ das freigesetzte Alizarin wie oben bestimmt. Als Kontrollversuch diente ein Ansatz mit Enzym und Ruberythrinsäure.

\section{Absorptionsspektren}

Alizarin und Ruberythrinsäure, deren Ausscheidungsprodukte vor und nach Spaltung mit $\beta$-Glucuronidase und Ruberythrinsäure nach Behandlung mit $\beta$-Glucuronidase wurden in $0,1 \mathrm{M}$ GlycinPuffer pH 11 gelöst und die Absorptionsspektren mit dem Spektralphotometer RPQ20A Zeiss im Bereich zwischen $800 \mathrm{~nm}$ und $450 \mathrm{~nm}$ geschrieben.

\section{Chromatographie}

1,5 mg Alizarin, 2,0 mg Ruberythrinsäure und 1,9 mg Fraktion I wurden in je $5 \mathrm{ml} 0,1 \mathrm{M}$ Glycin-Puffer $\mathrm{pH} 11,0$ gelöst. Davon trugen wir je $0,02 \mathrm{~m} l$, von den nach Spaltung mit $\beta$-Glucuronidase und Glycin-Pufferzugabe erhaltenen Lösungen $0,04 \mathrm{ml}$ auf Schleicher-Schüllpapier 2043b auf. Wir chromatographierten in dem oben angegebenen Fließmittel mit einer Laufzeit von $12 \mathrm{Stdn}$.

Ausscheidungsversuch

Drei Gruppen zu je zwölf männlichen Ratten, von denen immer zwei in einem Stoffwechselkäfig saßen, erhielten mit einer Schlundsonde je $6 \mathrm{~m} /$ Humanamilchzubereitung $(14 \mathrm{~g}$ Humanapulver Stufe I in $90 \mathrm{~m} l$ dest. Wasser gelöst). Zwei Stdn. später gaben wir mit der Schlundsonde der

Gruppe A: $6 \mathrm{~m} /$ Humana / Tier (Kontrolle)

Gruppe B: $6 \mathrm{~m} /$ Humana mit 9,6 mg Alizarin/ Tier

Gruppe C: $6 \mathrm{~m} /$ Humana mit 21,3 mg Ruberythrinsäure (Alizarinäquivalent).

Vier und acht Stdn. nach der Hydroxyanthrachinongabe erhielten alle Tiere weitere $6 \mathrm{~m} /$ Humana oral. In zweistündigen Abständen wurde 14 Stdn. lang der Harn gesammelt und gruppenweise zur Analyse vereinigt. Eine letzte Harnportion fingen wir 24 Stdn. nach der Hydroxyanthrachinongabe auf. In den einzelnen Harnportionen der drei verschiedenen Gruppen wurden Glucuronsäureund nach $\beta$-Glucuronidasespaltung Alizarinbestimmungen durchgeführt.

\section{Ergebnisse}

Identifizierung der im Harn ausgeschiedenen Hydroxyanthrachinone

\section{Alizarin- und Glucuronsäurenacbiveis}

a) Fraktion I nach Verfütterung von Alizarin

Fraktion I ist ein ziegelrotes amorphes Pulver, das im sauren Bereich mit gelber Farbe, im neutralen mit roter Farbe gut löslich ist. Alizarin und Glucuronsäure konnten im Verhältnis 1:1 nachgewiesen werden. Verglichen mit reinem Alizaringlucuronid haftete der gereinigten Fraktion I noch eine Verunreinigung von etwa $15 \%$ an. Eine nur einmal mit Alkohol gereinigte Fraktion I enthielt noch etwa $48 \%$ anthrachinonfreie Verunreinigungen.

\section{b) Fraktion II nach Verfütterung von Alizarin}

Es handelt sich um eine sirupartige Masse von schwach gelblicher Farbe. Sie besteht nur zu etwa $1 \%$ aus Alizarin und Glucuronsäure im Verhältnis 1:1. Nach Zusatz von $3 \mathrm{~N} \mathrm{NaOH}$ entwichen stechende ammoniakalische Dämpfe.

c) Fraktion I nach Verfütterung von Ruberythrinsäure Es handelt sich ebenfalls um ein ziegelrotes amorphes Pulver, das gleiche Löslichkeit zeigt wie Fraktion I nach
Verfütterung von Alizarin. In der nach einmaliger Alkoholbehandlung isolierten Fraktion I fanden wir 23\% Glucuronsäure und $25 \%$ Alizarin entsprechend einem 1:1-Glucuronid. Die Verunreinigung betrug etwa 52\%.

\section{Spaltung mit $\beta$-Glucuronidase}

Aus beiden Harnfraktionen I wurde innerhalb von sieben Min. das gesamte Alizarin mit gleicher Geschwindigkeit freigesetzt (Abb. 1). Aus reiner Ruberythrinsäure wurde in der gleichen Zeit kein Alizarin frei.

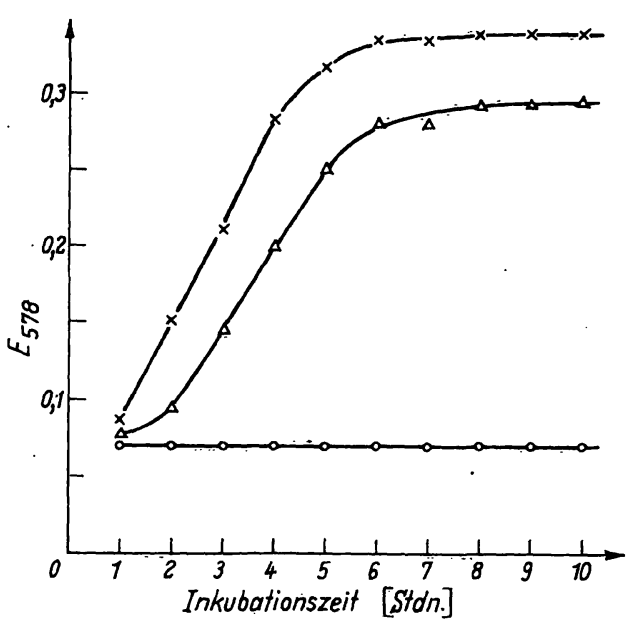

Abb. 1

Spaltung von Harnfraktion I bzw. Ruberythrinsäure durch $\beta$ Glucuronidase bei $37^{\circ}$. Gemessen wurde die Extinktion freigesetzten Alizarins

$x-x$ Harnfraktion I $158,3 \mu \mathrm{g} / 6,5 \mathrm{ml}$ isoliert nach Alizaringabe $\Delta-\Delta$ Harnfraktion I $223,4 \mu \mathrm{g} / 6,5 \mathrm{ml}$ isoliert nach Ruberythrin○- o Ruberythrinsäure $127,7 \mu \mathrm{g} / 6,5 \mathrm{ml}$

\section{Absorptionsspektren der verschiedenen Substanzen}

Beide Harnfraktionen I und Ruberythrinsäure zeigen identische Spektren mit einem Maximum bei $490 \mathrm{~nm}$ (Abb. 2, 4, 5).

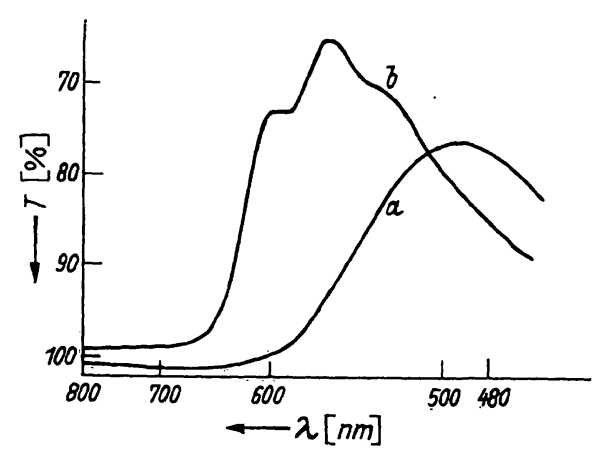

Abb. 2

Absorptionsspektren von Harnfraktion I und Alizarin a: Harnfraktion I, isoliert nach Alizaringabe. $3 \mu \mathrm{g} / \mathrm{m} l$ b: Alizarin, $1,2 \mu \mathrm{g} / \mathrm{m} l$

Alizarin besitzt dagegen ein typisches Maximum bei $578 \mathrm{~nm}$ (Abb. 2). Spaltet man die Harnfraktion I mit $\beta$-Glucuronidase, so erhält man wieder das typische Alizarinspektrum mit dem Maximum bei $578 \mathrm{~nm}$ (Abb. 3). Spaltet man Harnfraktion I, die nach Rub- 
erythrinsäuregabe isoliert wurde, mit $\beta$-Glucuronidase, so erhält man ebenfalls das Absorptionsspektrum des Alizarins (Abb. 4).

Inkubiert man Ruberythrinsäure mit $\beta$-Glucuronidase, so ändert sich das Maximum bei $490 \mathrm{~nm}$ während der Inkubationszeit von $10 \mathrm{Min}$. nicht (Abb. 5).

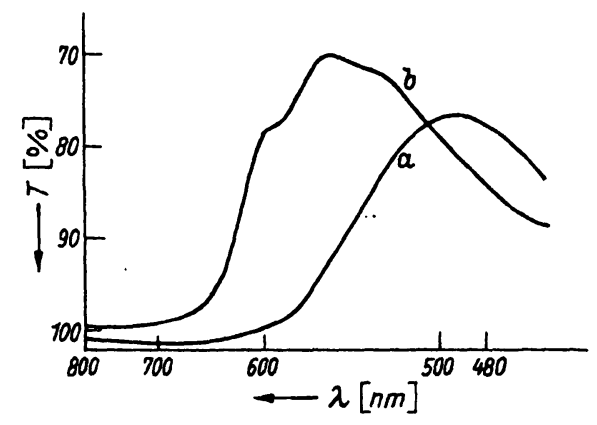

Abb. 3

Absorptionsspektren von Harnfraktion I vor und nach Inkubation mit $\beta$-Glucuronidase

a: Harnfraktion 1, isoliert nach Alizaringabe $3 \mu \mathrm{g} / \mathrm{ml}$.

b: Harnfraktion $I$, isoliert nach Alizaringabe, nach Inkubation mit $\beta$ Glucuronidase

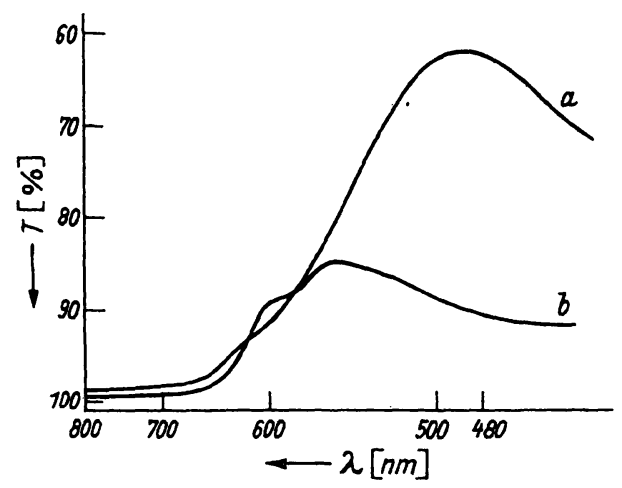

Abb. 4

Absorptionsspektren von Harnfraktion I vor und nach Spaltung mit $\beta$-Glucuronidase

a: Harnfraktion I, isoliert nach Ruberythrinsäuregabe $4 \mu \mathrm{g} / \mathrm{ml}$.

b: Harnfraktion I, isoliert nach Ruberythrinsäuregabe nach Spaltung mit $\beta$-Glucuronidase

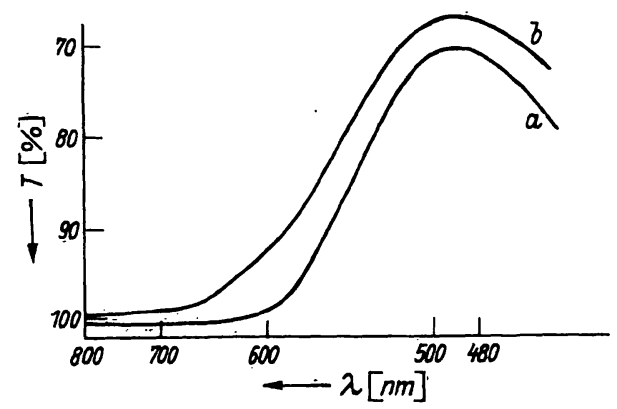

Abb. 5

Absorptionsspektren von Ruberythrinsäure vor und nach Inkubation mit $\beta$-Glucuronidase

a: Ruberythrinsäure $1,1 \mu \mathrm{g} / \mathrm{ml}$.
b: Ruberythrinsäure nach Inkubation mit $\beta$-Glucuronidase

\section{Chromatograplisie}

Die nach Alizarin- wie auch nach Ruberythrinsäuregabe isolierten Harnfraktionen I haben identische $R_{\mathrm{F}}$-Werte von 0,64. Ruberythrinsäure besitzt den $R_{\mathrm{F}}$-Wert von 0,83 . Spaltet man diese drei Stoffe $10 \mathrm{Min}$. lang mit $\beta$-Glucuronidase, so wird aus den beiden Harnfraktionen I Alizarin, das im Chromatogramm mit der Front läuft, freigesetzt. In der gleichen Inkubationszeit ändert sich $\operatorname{der} R_{\mathrm{F}}$-Wert von Ruberythrinsäure nicht.

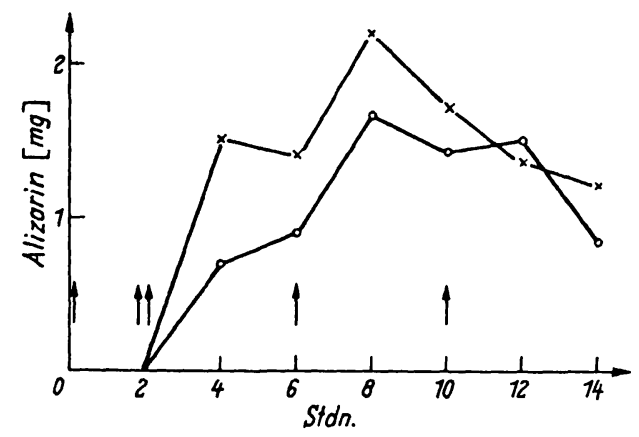

Abb. 6

Hydroxyanthrachinonausscheidung (als Alizarin) im Harn bei Ratten

$$
\begin{aligned}
& \because-\because \text { Alizarin } 115,1 \mathrm{mg} / 12 \text { Ratten } \\
& \circ \text { Ruberythrinsäure } 256 \mathrm{mg} / 12 \text { Ratten } \\
& \uparrow: 6 \mathrm{ml} \text { Humana } \\
& \uparrow: 6 \mathrm{~m} l \text { Humana + Hydroxyanthrachinone }
\end{aligned}
$$

Ausscheidungsversuche

Nach Verfütterung von insgesamt $115,2 \mathrm{mg}$ Alizarin an 12 Ratten wurden im Harn nach Hydrolyse innerhalb von 12 Stdn. 9,49 mg wiedergefunden (Abb. 6). Dies entspricht $8,24 \%$. Nach weiteren $12 \mathrm{Stdn}$. wurden noch $2,44 \mathrm{mg}$ ausgeschieden, so daß innerhalb von $24 \mathrm{Stdn}$. $10,38 \%$ der oral zugeführten Menge an Alizarin im Harn wiedergefunden wurde. Nach Gabe einer äquivalenten Menge Ruberythrinsäure wurden im Harn innerhalb von $12 \mathrm{Stdn} .7,15 \mathrm{mg}$ Alizarin gefunden. Dies entspricht $6,2 \%$ der verabfolgten Dosis. In den nächsten 12 Stdn. wurden weitere $1,87 \mathrm{mg}$ Alizarin ausgeschieden, so daß von der Gesamtmenge innerhalb von 24 Stdn. 7,82\% ausgeschieden wurden. Das Maximum der Glucuronsäure- (Abb. 7) wie auch der Alizarinausscheidung ist 6 Stdn. nach der Hydroxyanthrachinongabe erreicht.

\section{Diskussion}

Glucuronsäure spielt bei der Elimination von körpereigenen wie auch körperfremden Substanzen eine wichtige Rolle. Die von uns nach Alizarinverfütterung als Fraktion I isolierte Substanz enthält Glucuronsäure und Alizarin im Verhältnis 1:1. Wir nehmen ein Alizarinmonoglucuronid an. Chromatographisch und mit Hilfe von Absorptionsspektren konnte bewiesen werden, $\mathrm{da} \beta$ durch Inkubation mit $\beta$-Glucuronidase aus der Fraktion I tatsächlich Alizarin freigesetzt wird.

$\mathrm{Da}$ das Alizaringlucuronid das gleiche Absorptionsspektrum wie die Ruberythrinsäure aufweist, deren Kohlenhydrat-Komponente in Position 2 steht, darf man 


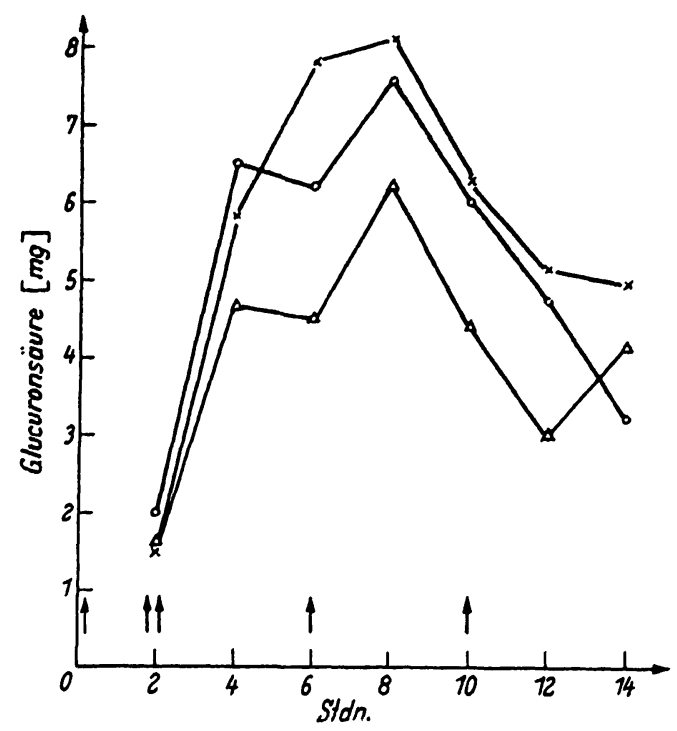

Abb. 7

Glucuronsäurcausschcidung im Harn nach Hydroxyanthrachinongabe bei Ratten

\begin{tabular}{l}
$:-:$ Alizarin $115,1 \mathrm{mg} / 12$ Ratten \\
\hdashline Ruberythrinsäure $256,3 \mathrm{mg} / 12$ Ratten \\
$i-\therefore$ Kontrolle 12 Ratten \\
$i: 6 \mathrm{ml}$ Humana \\
$i 1: 6 \mathrm{ml}$ Humana + Hydroxyanthrachinone
\end{tabular}

annchmen, daß auch dic Glucuronsäure ätherartig an die OH-Gruppe in Stellung 2 gebunden ist. Dies steht im Einklang rnit der Tatsache, daß die OH-Gruppe in Stellung 1 eine starke Nebenvalenzbindung zu der benachbarten Oxogruppe besitzt (6).

Nach den vorliegenden Ergebnissen ist es sicher, daß auch die Ruberythrinsäure nach oraler Gabe als Alizarinmonoglucuronid im Harn erscheint. Aus Ruberythrinsäure läßt sich innerhalb von 10 Min. durch Inkubation mit $\beta$-Glucuronidase kein Alizarin freisetzen, während dics bei der Fraktion I, isoliert nach Ruberythrinsäure- gabe, der Fall ist. Es kann sich also bei der verfütterten Ruberythrinsäure und ihrem Ausscheidungsprodukt nicht um die gleiche Substanz handeln. Beide Substanzen haben einen verschiedenen $R_{\mathrm{F}}$-Wert. Die Fraktion I, gewonnen nach Ruberythrinsäuregabe, ist dagegen mit der Fraktion I, isoliert nach Alizarinverfütterung, identisch. Beide Harn-Fräktionen I zeigen gleiche Eigenschaften gegenüber der Behandlung mit $\beta$-Glucuronidase, beide besitzen identische $R_{\mathrm{F}}$-Werte. Das Verhältnis von Glucuronsäure zu Alizarin wurde in der nach Ruberythrinsäuregabe isolierten Fraktion I zu 1:0.9 bestimmt. Die nur einmal mit Alkohol aus Harn extrahierte Substanz ist wie nach Alizaringabe etwa $50 \%$ rein.

Bei der Harn-Fraktion II handelt es sich wahrscheinlich um ein nicht völlig von Alizaringlucuronid gereinigtes primäres Amin. Derartige Amine, z. B. Methylamin, sind im Rattenharn als basensparendes Prinzip bekannt.

$\mathrm{DaB}$ rund $10 \%$ der Hydroxyanthrachinone nach Alizarin und rund $8 \%$ nach Ruberythrinsäureverfütterung innerhalb von 24 Stdn. im Harn wiedererscheinen, deckt sich mit Ergebnissen von BODE (8), die nach Applikation von Hydroxybenzoesäuren eine Erhöhung der Glucuronsäureausscheidung um rund 10 bis $15 \%$ gegenüber den Kontrollen beobachtete. Die restlichen $90 \%$ der Hydroxyanthrachinone könnten entweder nicht resorbiert worden, teilweise im Knochen fixiert oder mit der Galle ausgeschieden sein. ERBRING und Mitarbeiter (9) haben chromatographisch bestätigt, daß auch beim Menschen nach oraler Gabe von Alizarin und Ruberythrinsäure im Harn Alizaringlucuronid erscheint. Rohe Ruberythrinsäurepräparationen führten zu stärkerer Alizaringlucuronidausscheidung als reines Alizarin. Ruberythrinsäure tritt im Harn des Menschen nach Belastung mit Hydroxyanthrachinonen ebenfalls nicht auf (9).

Wir danken der Fa. Dr. Madaus für die freundliche Uberlassung chromatographisch cinheitlicher Ruberythrinsäurc.

\section{Literatur}

1. Dulce, H:-J., Unveröffentl. Mitteilung. - 2. Madaus, G. und E. KoCH, Zschr. Exp. Med. 109, 517 (1941). - 3. KrLLER, J. und B. GörliCII, Zschr. Urol. 38, 1 (1944). - 4. BAUER, A., Zschr. Urol. 14, 175 (1920). - 5. BAUER, A., Zschr. Urol. 17, 274 (1923).

- G. Hais, I. M. und K. MAČek, Handb. der Papierchromato- graphie, Bd. I., S. 329 (1963) VEB Gustav Fischer Verlag Jena. 7. Drsche, Z., J. biol. Chemistry 167, 191 (1947). - 8. Bode, E., Inaug. Dissert. Bcrlin (1961). - 9. Erbring, H., Persönl. Mittcilung.

Prof. Dr. H.-J. Dulce 1 Berlin 33

Arnimallee 22 